\title{
THE ABSORPTION OF HYDROGEN BY METALLIC FILMS.
}

By Will C. Baker.

PROFESSOR SKINNER ${ }^{1}$ has shown that when a discharge is sent, at low gas pressure, to a freshly polished cathode, hydrogen is liberated from the metal initially in the quantities required by Faraday's law for electrolytes. The proportionality between the mass of gas set free per second and the current soon diminishes (if the current be maintained), presumably as the available hydrogen in the surface layers is exhausted; for on standing in a hydrogen-free atmosphere the metal recovers its original power of giving off gas, probably through diffusion from the deeper layers. Heald, ${ }^{2}$ in attempting to measure this absorbed gas by volatilizing the metal in a partial vacuum, concluded that the films thus condensed on the walls of the tube showed a measurable absorption of hydrogen. He states that even with quantities of active metal "too small to measure," a diminution of pressure was observable for an hour or more after the production of the film. Skinner ${ }^{3}$ had already shown that the cathode in his experiments did not re-absorb the gas that had been liberated from it; but this was possibly due to the more ready absorption of hydrogen (probably atomic) from the interior of the metal. Thus it appeared quite possible - especially in view of Heald's observations - that a film of metal produced in a hydrogen-free atmosphere might show a measurable absorption on being exposed to hydrogen; also that it might be possible to determine the quantity of gas absorbed per gram of metal, if it were a volume effect; or the quantity of gas condensed per unit area if it were merely a surface phenomenon.

To decide these points metallic films of measurable mass were deposited by "spluttering" from a cathode, through a gas that had

1 Phys. Rev., July, 1905.

2 Phys. Rev., March, I907.

${ }^{3}$ Loc. cit. 
been carefully freed from hydrogen. The apparatus was then allowed to stand for an hour to permit it to cool from any heating arising from the deposition of the film; and finally, a known quantity of hydrogen was forced into the apparatus and the pressure measured from time to time. As the volume employed was less than one tenth of that used by Heald, and as weighable quantities of metal were used, the effect, if it existed under these conditions, would have been easily measurable. It was usually one minute after the introduction of the hydrogen that the first pressure reading was taken; but the pressure obtaining at the first contact of the film

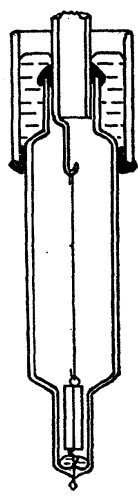

Fig. 1 .

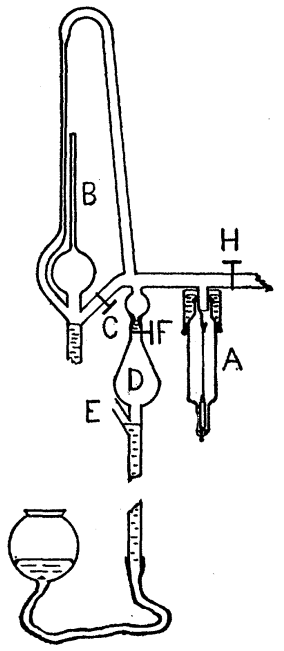

Fig. 2.

and gas could be approximately found from the volume of the apparatus, the pressure before the injection of the hydrogen, and the quantity of that gas used. Thus any absorption occurring in the first minute would have been detected. Cathodes of silver, iron and platinum were used. The two former are metals that gave large values in Heald's experiments. In no case was any absorption detected.

Figs. I and 2 give a diagrammatic representation of the experimental arrangements. In order to increase pressure changes the apparatus was kept as small as convenient, the main chamber with 
attached gauge, etc., had a volume of about forty cubic centimeters. The cathode consisted of a fine wire seven centimeters long. This was hung from a glass hook as shown in Fig. I and was easily weighable before and after the experiment. It was slightly stretched by supporting at its lower end a small piece of capillary tube through which passed an aluminium ${ }^{1}$ wire, making connection between the cathode and the fused-in platinum lead through a drop of mercury. This discharge chamber was attached to the rest of the apparatus by a sealing-wax joint, "drowned" in mercury; the latter serving to give it heat capacity, thus preventing the wax from softening during the discharge. The film had a superficial area of about forty square centimeters. The relation of this tube to the rest of the apparatus is shown in Fig. 2. $A$ is the discharge chamber. $B$ is a McLeod gauge. In order to enable readings to be made quickly, a by-pass with a tap, $C$, connected the main apparatus to the stem of the gauge at a point about a millimeter below the level of the capillary side tube. As this by-pass was about four millimeters diameter, the gauge quickly took up the pressure of the apparatus when the tap was open. In taking a reading the tap, $C$, was closed just after the mouth of the by-pass was covered by the rising mercury. The gauge then acted in the usual way. This arrangement gave complete satisfaction. The chamber, $D(54.5$ cc.), with $\operatorname{tap} F$, served to give a definite volume of hydrogen and was connected through $E$ to a half liter reservoir where that gas was stored over $\mathrm{P}_{2} \mathrm{O}_{5}$. A second McLeod gauge, also in connection with $D$, gave the pressure of this part of the apparatus. A fiducial mark was made about a centimeter above $F$, at a constriction in the tube, and the volume between this and $F$ was kept full of mercury, forming a seal against hydrogen leaking into the discharge chamber from $D$. Knowing the volume of $D$ from the side tube $E$, to $F$; and adjusting the pressure of the hydrogen filling it, a definite quantity of gas could be sent into the apparatus by first raising the mercury so as to close $E$; opening $F$, thus letting the mercury seal fall into $D$; then on continuing to raise the mercury to the fiducial mark the gas was forced into the main apparatus and the tap sealed as before. Between the tap $H$, which limited the

'Aluminium does not "splutter", in vacuo. 
No. 5.] ABSORPTION OF HYDROGEN BY METALLIC FILMS. 425

experimental volume in that direction, and the pump, were attached (I) a bulb containing the liquid alloy of sodium and potassium, (2) a spectrum tube an electrode of which served as the anode of the discharge chamber, and (3) a drying bulb with $\mathrm{P}_{2} \mathrm{O}_{5}$. The gauges "magnified" the pressure fifty times. All the taps were lubricated with a mixture of paraffin, vaseline and rubber according to Travers" formula.

The experimental procedure was as follows: The discharge chamber and cathode were removed and a closed tube sealed on in their place. The $\operatorname{tap} F$ being open, the entire apparatus was exhausted by a water pump ; then hydrogen ${ }^{2}$ was let in through a tap not shown. This exhaustion and filling was repeated four or five times. The whole apparatus was next exhausted by the Toepler pump to a pressure of about one millimeter. The mercury was raised, filling $D$, and its level adjusted to the fiducial mark. $F$ was then closed, and air admitted to the main apparatus. A current of air, entering through the stem of gauge $B$ was maintained until the hydrogen had been swept out of the gauge and pump. The discharge chamber and weighed cathode were next put in place and the apparatus filled with nitrogen. ${ }^{3}$ In the cases of iron and platinum the cathode was made red hot in a nitrogen atmosphere by running the discharge, at a pressure of six one hundredths of a millimeter; and the apparatus was filled with nitrogen four times more, exhausting each time to one millimeter. This gas was allowed to stand for twenty or twenty-two hours in contact with $\mathrm{P}_{2} \mathrm{O}_{5}$. After drying, the gas was purified from any remaining hydrogen by running the NaK bulb with the alloy as cathode, until the spectrum of hydrogen had entirely disappeared. A Browning direct vision spectroscope was used. After purification, the film was deposited, let cool for an hour, the hydrogen injected and the readings made. A few hours later the ratio of the volume of $D$ to the experimental volume was determined by measuring the increase in pressure produced in the apparatus by the introduction of a definite quantity of gas. This ratio varied from experiment to experiment due to the

1 The Study of Gases.

${ }^{2}$ From $\mathrm{Zn}$ and $\mathrm{HCl}$, dried by bubbling through strong $\mathrm{H}_{2} \mathrm{SO}_{4}$.

3 Prepared as recommended by Travers (loc. cit.) by drawing air from outside the building, through strong $\mathrm{NH}_{3}$, then over red hot copper turnings, and finally through two wash bottles of concentrated $\mathrm{H}_{2} \mathrm{SO}_{4}$ to remove moisture and excess of ammonia. 
fact that the sealing-wax joint was seldom twice in exactly the same place.

On account of the small volume employed the well-known effect of the lowering of the gas pressure accompanying discharge was especially prominent during the deposition of the film. In the case of iron five hours were required in this process. Also the $\mathrm{NaK}$ alloy absorbs nitrogen as well as hydrogen. This would soon

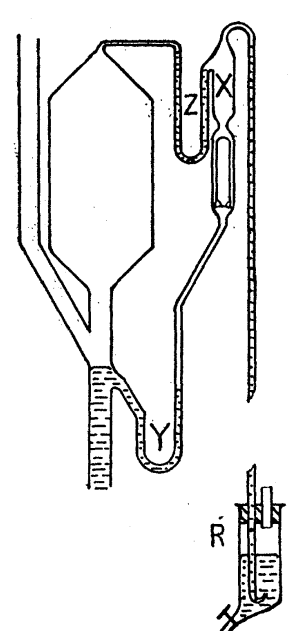

Fig. 3. increase the vacuum until the discharge would not pass were there not some means of feeding gas back into the tube. To overcome this the valve chamber of the pump was utilized. During the final drying the gas was left at a pressure of about a half a millimeter; the mercury having been turned completely through the pump leaving no gas in the valve chamber $X$ (Fig. 3). After drying and purifying, the apparatus was pumped down to the required pressure but the excess of gas was not turned out of the pump; it was allowed to remain in the chamber $X$, being kept there by the traps at $Y$ and $Z$. If now the mercury in the main stem were lowered below the level of the mouth of the tube $Y$, and if more mercury were poured into the fixed reservoir at $R$ until its surface was less than the barometric height below the bend above the valve chamber, large drops would fall on top of the open valve and slipping down would enclose gas in the tube $Y$ and force it over into the pump head and so into the apparatus. This process could be stopped by lowering the mercury in $R$. In this way dried and purified gas could be returned to the discharge tube at will.

Typical Measurements. Iron.

Cathode wire well cleaned with sand paper. Heated to bright redness in nitrogen at $4 / 50 \mathrm{~mm}$. Apparatus refilled four times.

Film deposited at $4 / 50 \mathrm{~mm}$. after drying and purifying gas. Film a light brown at edges but a black mirror in body of deposit.

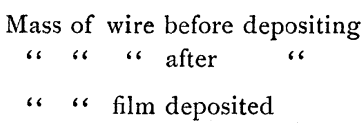

0.0280 gms. 0.0260 “

0.0020 gms. 
No. 5.] ABSORPTION OF HYDROGEN BY METALLIC FILMS. 427

$54.5 \mathrm{cc}$. hydrogen admitted to discharge chamber at Gas in discharge chamber before admission of hydrogen at Hydrogen injected at $11 \mathrm{hrs} .06 \mathrm{~min}$. A. M.

Pressure observed at 11 " 07 “

“ 08 “

" 10 " "

- “ 14 " "

“ 18 ،

“ 22 "

“ 27 ،

“ 38 “

“ 45 “

1 hr. 15 “ P. M.

Ratio of volume $D$ to discharge chamber, etc., 1.29.

Pressure immediately after injection of hydrogen, calculated,
32.5 fiftieths of $\mathrm{mm}$.

2.4 ، “ ،

45.2 fiftieths of $\mathrm{mm}$.

45.2 " " " "

45.2 " " " "

45.2 ، “ ،

45.1 " ، " ،

45.2 ، " ، " "

45.2 " " ،

45.2 “ ، ،

45.2 " " ،

45.0 “ ، “

\section{Silver.}

Wire cleaned with sand paper. Film deposited at $3 / 50 \mathrm{~mm}$. after drying and purifying gas. Film a bright metallic mirror.

\section{Mass of wire before depositing \\ " " " after \\ " " film deposited}

$54.4 \mathrm{cc}$. hydrogen admitted at

Gas in discharge chamber before admission of hydrogen at 2.4 " " "

Hydrogen injected at 9 hrs. 54 min. A. M.

Pressure observed at " 55 "

" 56 " "

" 58 " "

10 " 00 "

“ 03 “

" 06 " "

" 10 "

" 14 “

“ 19 "

“ 25 "

" 41 "

11 " 20 “"

1 hr. 04 " P. M.

2 hrs. 35 “"

0.0756 gms.

0.0744 “

0.0012 gms. 44.8 fiftieths of $\mathrm{mm}$
61.0 fiftieths of $\mathrm{mm}$.

61.0 " " " "

60.8 ، " " "

60.8 ، " ،

60.8 ، " ،

60.9 " ، " "

60.9 ، ، “

60.9 ، " " "

60.8 ، ، ،

60.9 ، ، " ،

60.9 ، " ، "

60.8 ، " ،

60.8 ، " ،

، ، ،
61.0 ، " ، " ،

9 " 00 " A. M. (next day) 61.8

Ratio of volume of $D$ to discharge chamber, etc., 1.29.

Pressure immediately after injection of hydrogen, calculated 60.2 " " " 
Platinum.

Cathode boiled in strong nitric acid to remove occluded hydrogen, washed, and dried in filter paper. After first filling with nitrogen the wire was raised to a bright red heat for one minute. During the deposition of the film the current was run to the wire for five seconds then to the NaK alloy for the next five seconds, then to the wire again and so on using the alloy and wire alternately, five seconds each, during the entire period of formation of the film. No trace of the hydrogen spectrum could be detected under this treatment. In other experiments it was found that after the gas had been purified, one could just detect the hydrogen lines when the discharge had run twenty seconds continuously. The film was a mirror, slightly dull owing to the deposition of larger particles during the heating after first filling. Film deposited at 3/50 mm.

\begin{tabular}{|c|c|c|c|c|c|c|c|c|}
\hline \multicolumn{4}{|c|}{ Mass of wire before deposit } & & \multicolumn{4}{|c|}{0.0261 gms. } \\
\hline$" 4$ & after & & \multicolumn{2}{|l|}{ “ } & \multicolumn{3}{|c|}{0.0243} & \\
\hline \multicolumn{5}{|c|}{ “ " film deposited } & \multicolumn{4}{|c|}{0.0018 gms. } \\
\hline \multirow{2}{*}{\multicolumn{5}{|c|}{$\begin{array}{l}54.5 \mathrm{cc} \text {. hydrogen admitted at } \\
\text { Gas in discharge chamber before admission of hydrogen at } \\
\text { Hydrogen injected at. } 11 \text { hrs. } 20 \mathrm{~min} \text {. A. M. }\end{array}$}} & \multicolumn{4}{|c|}{38.2 fiftieths of $\mathrm{mm}$} \\
\hline & & & & & 1.2 & “6 & “6 & “ \\
\hline \multirow[t]{9}{*}{ Pressure observed at } & & “6 & 21 & "6 & \multicolumn{4}{|c|}{51.7 fiftieths of $\mathrm{mm}$. } \\
\hline & & & 23 & "، & 51.7 & “ & 66 & "6 \\
\hline & & “" & 25 & “6 & 51.7 & “6 & “ & 6 \\
\hline & & & 29 & “6 & 51.7 & “6 & “ & \\
\hline & & “ & 34 & “6 & 51.7 & “ & “ & 6 \\
\hline & & “ & 40 & “6 & 51.7 & “6 & “ & 6 \\
\hline & & “ & 45 & “" & 51.7 & "6 & “ & 6 \\
\hline & 12 & “6 & 50 & 6 & 51.7 & “6 & "6 & 6 \\
\hline & & hr. & 45 & " P. M. & 51.7 & “6 & 66 & 6 \\
\hline
\end{tabular}

The measurements given above indicate that cold metallic films, resulting from cathode deposition, across a hydrogen-free space, do not show a rapid absorption of hydrogen from the molecular state, at least within the limits of accuracy of the experiments recorded in this note.

Physical Laboratory, School of Mining, Queen's University, Kingston, ONT.

1 Wilson, H. A., Phil. Trans. Roy. Soc., A, Vol. 202, p. 253. 